MODELING, IDENTIFICATION AND CONTROL, 1999, vOL. 20, NO. 4, 225-240

doi:10.4173/mic.1999.4.3

\title{
Stabilization of a Pitch Axis Flight Control Experiment with Input Rate Saturation*
}

\author{
TRYGVE LAUVDAL $\dagger$ and RICHARD M. MURRAY $\ddagger$
}

Keywords: Rate saturation, nonlinear control, ducted fan

\begin{abstract}
Motivated by some specific problems in flight control and roll stabilization of ships, we present a technique for stabilizing a chain of integrators in the presence of rate limitations on the input. Our technique improves on several existing techniques in the literature and has a number of interesting features. The controller is evaluated experimentally on a pitch axis flight control experiment at Caltech. The experimental results show that even in the presence of rate limits that cause a linear controller to go unstable, the time-varying controller stabilizes the system with minimal loss in performance.
\end{abstract}

\section{Introduction}

One of the most significant sources of nonlinearities in control systems is actuator saturation, which occurs in virtually all modern systems but is widely ignored by the existing analysis and synthesis tools. At present, there is no systematic means of analyzing and designing nonlinear control systems in the presence of magnitude and rate saturations.

One application area in which saturation nonlinearities are particularly prevalent is flight control of high agility aircraft, where actuator saturation has a significant effect on the overall stability of aircraft. A specific instance is the YF-22 crash of April, 1992, which has been blamed on a pilot-induced oscillation (PIO) caused in part by time-delay effects introduced by rate saturation of control surfaces (Buchholz 1993; Dornheim 1992). As the complexity and performance of flight systems increase, stronger theoretical understanding is required to avoid such situations and guarantee performance of the system in the face of noise and unmodeled dynamics.

At the practical level, saturation in most systems is handled in an ad hoc fashion. Gains are chosen and artificial saturations are inserted such that the system performs well in simulations and experimental tests. Rate saturations are sometimes modeled as equivalent time-delays to allow the use of linear control theory (Buchholz 1993). While these techniques work for systems of low dimension and reasonably straightforward dynamics, as systems get more complicated the difficulties in this ad hoc approach become more noticeable (as in the crash of the YF-22). Further demands for increased performance and agility will exacerbate this problem.

Many techniques are available in the linear literature for incorporating actuator saturations into the design process. One example is the use of $l_{1}$ analysis and synthesis techniques, which allow specification of the maximum output response as a function of the maximum size of noise and disturbances (Dahleh and Pearson 1987; Dahleh

${ }^{*}$ Originally presented at AIAA Guidance, Navigation and Control Conference, New Orleans, August 1997.

$\dagger$ ABB Industri AS, Oslo.

$\ddagger$ Assistant Professor, California Institute of Technology. 
and Diaz-Bobillo 1995). Other techniques include the use of convex optimization to design controllers with a variety of input and performance constraints (Boyd and Barratt 1991). A major limitation of these approaches is that they only work for linear systems and they generate linear controllers. As a result, the designs can be very conservative (since the gain must be small enough to tolerate the worst case scenario) and it may be difficult to achieve high performance.

Several new nonlinear tools have been introduced in the last four years for analyzing and controlling linear and nonlinear systems with saturation. One of the fundamental techniques is based on the thesis work of Teel (1992a,b), who showed how to stabilize a chain of integrators using nested saturation functions. This result is significant since it is known that it is not possible to stabilize a chain of three or more integrators using a linear control law followed by a saturation function. Thus even simple linear systems with simple saturations can give rise to difficult nonlinear problems. Teel's approach generates nonlinear controllers which are locally linear, but become nonlinear as the inputs grow toward the saturation limits.

Another approach has been introduced by Megretski (1996), who uses a gain scheduling approach to generate nonlinear stabilizers for saturated linear systems. In addition to stability, Megretski shows that for stable plants the map from plant disturbances to control inputs is $L_{2}$ bounded.

In this paper we present a specific technique for stabilizing a chain of integrators in the presence of rate saturations. The approach which we develop gives a provably convergent closed-loop system (see Lauvdal et al. (1997) for details) and very good performance compared to other methods proposed in the literature. Although the scope of the solution presented here is limited to a chain of integrators, the basic techniques and ideas serve as a starting point for a more general approach to control of nonlinear systems in the presence of actuator bandwidth, magnitude, and rate limitations.

This paper is organized as follows. Section 2 gives a short problem description. Section 3 presents the gain scheduling method used and in Section 4 the scheduling factor is derived. A simulation study and experimental results on the Caltech ducted fan are presented in Section 6 and Section 7.

\section{Problem Description}

The problem we consider in this paper is to stabilize a chain of integrators

$$
\begin{gathered}
\dot{x}_{1}=x_{2} \\
\dot{x}_{2}=x_{3} \\
\vdots \\
\dot{x}_{n}=u
\end{gathered}
$$

where $x_{i} \in \mathbb{R}, i=1, \ldots, n$ are the states and $u \in \mathbb{R}$ is the input and is subject to input nonlinearities. Usually, input nonlinearities are required to be a memory-free saturation type function, that is, it resides in the first and third quadrants and satisfy some sector conditions. In this paper we derive a stabilizing controller for nonlinearities with memory, and in particular we consider the case of rate saturation. Hence, the input dynamics is given by

$$
\dot{u}=\operatorname{sat}\left(\frac{1}{\tau}\left(u_{c}-u\right)\right)
$$


where $u_{c} \in \mathbb{R}$ is the commanded input and the function sat: $\mathbb{R} \rightarrow[-\beta, \beta]$ is a saturation function given by

$$
\operatorname{sat}(\cdot) \triangleq \operatorname{sign}(\cdot) \min (|\cdot|, \beta), \beta>0
$$

The parameter $\tau$ describes the linear part of the input dynamics and is assumed to be small since the focus of this paper is on the destabilizing effect of a rate saturated signal. Hence, the linear dynamics can be neglected and the saturation function is considered to be a relay type function, denoted $\sigma$. Then, if $(A, b)$ describes the integrator chain (1), the system can be written in the compact form

$$
\begin{aligned}
& \dot{\boldsymbol{x}}=\boldsymbol{A x}+\boldsymbol{b} u \\
& \dot{u}=\sigma\left(u_{c}-u\right)
\end{aligned}
$$

where $\boldsymbol{x} \in \mathbb{R}^{n}$ and $u, u_{c} \in \mathbb{R}$.

The goal of the control design in this paper is not only to make the closed-loop system convergent, but also to obtain a control system with good performance. With good performance we mean

- A rise time as close as possible to the rise time of the linear system with linear controller.

- No large overshoot compared to the response of the linear system with linear controller (also called the 'anti-windup' problem).

Convergence of the closed-loop system is not very hard to obtain on its own. It has been shown (Lauvdal and Fossen 1997) that for all null-controllable systems, and thus in particular for integrator chains, subject to input rate saturation, there exists a linear state feedback such that the closed-loop system is locally convergent. However, like any other linear control law it will in general be very conservative since the control gains must be small enough to tolerate the worst case scenario.

\section{Gain Scheduling Method}

In order to stabilize the system (4) we take a gain scheduling approach and a key feature of the results presented in this paper is the gain scheduling method. Assume we have designed a linear controller given by

$$
u_{c}=-\boldsymbol{g}^{T} \boldsymbol{x}
$$

which stabilizes the system (4) when assuming $u=u_{c}, t \geqslant t_{0}$. Then the gain scheduled control law is defined as follows.

Definition 1. Let $u_{c}=-g^{T} x$ be a linear control law stabilizing the pair $(A, b)$ under the assumption $u=u_{c}$. Then, the gain scheduled control law is defined to be

$$
u_{c}(t, x) \triangleq-g^{T} K(\gamma(t)) x
$$

where

$$
K(\gamma(t))=\operatorname{diag}\left(\gamma^{n}(t), \ldots, \gamma(t)\right)
$$

and $\gamma(t) \in(0,1]$ is the scaling factor.

Remark 1. Notice that the scaling factor $\gamma(t)$ is a function of time. This is essential in order to obtain a controller which is not overly conservative. 
Consider the case when the scaling factor is fixed, $\gamma(t) \equiv \gamma$. Then, applying the resulting linear controller to the integrator chain (4), the closed-loop system matrix is given by

$$
A_{c}(\gamma)=\left(A-b g^{T} K(\gamma)\right), \quad \gamma \in(0,1]
$$

and Definition 1 implies the following characteristics on the closed loop system.

Proposition 1. Assume that $A_{c}(1)$ is a Hurwitz matrix. Then, $A_{c}(\gamma)$ is Hurwitz for each fixed $\gamma \in(0,1]$.

Proof: Let $\lambda_{i}, \mathrm{i}=1, \ldots, n$, denote the eigenvalues of $A_{c}(1)=\left(A-b g^{T}\right)$. Then it can be shown that the eigenvalues of $A_{c}(\gamma)=\left(\boldsymbol{A}-\boldsymbol{b} \boldsymbol{g}^{T} \boldsymbol{K}(\gamma)\right)$ are given by

$$
\lambda_{i}(\gamma)=\gamma \lambda_{i}, \quad i=1, \ldots, n
$$

Hence, if $\lambda_{i}, i=1, \ldots, n$, are in the open left-half part of the complex plane, then so are $\lambda_{i}(\gamma), i=1, \ldots, n$, for each fixed $\gamma \in(0,1]$.

In order to get better insight into the properties of the scheduled control law and to illustrate the reason for the nonlinear scaling as opposed to linear scaling, consider the following example.

Example 1. Consider an integrator chain of length 4, i.e.

$$
\dot{x}_{1}=x_{2}, \dot{x}_{2}=x_{3}, \dot{x}_{3}=x_{4}, \dot{x}_{4}=u
$$

with a linear stabilizing controller $u=-g^{T} x$, where

$$
g=\left[\begin{array}{llll}
1.0000 & 3.0777 & 4.2361 & 3.0777
\end{array}\right]^{T}
$$

Thus, the closed-loop system with fixed scaling of the input becomes

$$
A-b g^{T} K(\gamma), \quad \gamma \in[0,1]
$$

for some $\boldsymbol{K}(\gamma)$. First, consider linear input scaling, i.e. $\boldsymbol{K}(\gamma)=\gamma \boldsymbol{I}$. The eigenvalues of (12) as function of $\gamma$ are shown in the left plot of Figure 1, and it is seen that linear
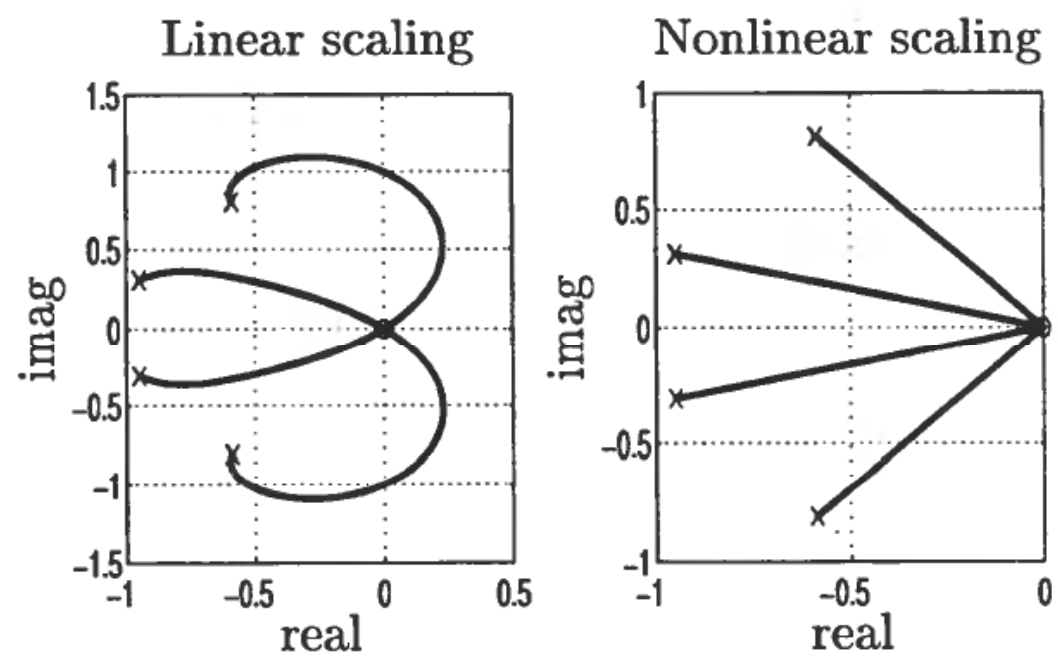

Figure 1. Illustration of the closed-loop eigenvalues as function of $\gamma$ for the linear (left plot) and nonlinear (right plot) scaling methods. ' $x$ ' indicates the nominal closed-loop eigenvalues $(\gamma=1)$ and 'o' the open-loop eigenvalues $(\gamma=0)$. 
scaling can lead to eigenvalues with positive real part. Next, consider nonlinear scaling of the input and let $K(\gamma)$ be given by (7). Then, from the right plot of Figure 1 it is seen that the eigenvalues of (12) have negative real parts for $\gamma \in(0,1]$. In fact, the damping ratio is kept constant since the eigenvalues are moving linearly towards the origin.

Remark 2. It should be noted that integrator chains of dimension $n \leqslant 2$ will have closed-loop eigenvalues with negative real parts also in the case of linear scaling. For $n>2$ the linear scaling will in general give closed-loop eigenvalues with positive real parts.

In the following section we will derive a particular scaling factor $\gamma(t)$ which have some interesting properties.

\section{Scaling Factor}

Given the scaling method presented above, a scaling factor needs to be found. In order to obtain a closed-loop system satisfying the performance criteria, we make the scaling factor a function of time rather than a function of the states. Then, the first question is when to reduce the scaling factor, $\gamma(t)$. We know that for a linear system with a linear controller subject to input rate saturation, there exists a set $\chi \subset \mathbb{R}^{n}$ such that if $\boldsymbol{x}\left(t_{0}\right) \in \chi$ the state of the closed-loop system converges to the origin. Thus, if $h(t)$ denotes the time for which $u_{c}>u$ or $u_{c}<u$, i.e.

$$
h(t)=0 \text { if } u=\mathrm{u}_{c}, \quad \dot{h}(t)=1 \text { otherwise }
$$

it is clear that there exists a limit, $\bar{h}$, such that $h(t) \leqslant \bar{h}$ for all $x\left(t_{0}\right) \in \chi$. Based on this observation, we propose to introduce some threshold, $\Delta T>0$, and reduce the $\gamma(t)$ when $h(t)>\Delta T$. Hence,

$$
\dot{\gamma}(t)<0 \text {, if } h(t)>\Delta T
$$

To illustrate the intuition behind this approach, consider the following example.

Example 2. Consider a system of two integrators

$$
\dot{x}_{1}=x_{2}, \dot{x}_{2}=u
$$

with a linear stabilizing controller $u=-g^{T} x$, where

$$
g=\left[\begin{array}{ll}
1.000 & 1 \cdot 7321
\end{array}\right]^{T}
$$

The input is subject to rate saturation of unity value, $\beta=1$, and it is assumed that $u(0)=0$.

First, we are interested in the maximal set of initial conditions $\chi$ such that for all $\boldsymbol{x}(0) \in \chi$ the state converges to the origin. An estimate was found through simulations and is shown in Figure 2 (solid line). This set can also be characterized in terms of $h(t)$, since $h(t)$ is bounded from above by $\bar{h}=3.8$ for all $x(0) \in \chi$. Hence, if $\Delta T=\bar{h}$ the controller will remain linear for all $x(0) \in \chi$.

It is not always desirable to take $\Delta T$ as large as possible, i.e. $\Delta T=\bar{h}$. This is illustrated by the time response for $\boldsymbol{x}(0)=[5,-3 \cdot 27]^{T} \in \chi$, shown in Figure 2 (dashed line). It is seen that the states oscillates before converging to the origin (the control system exhibit 'windup'). This is caused by the rate saturated input, and by lowering 


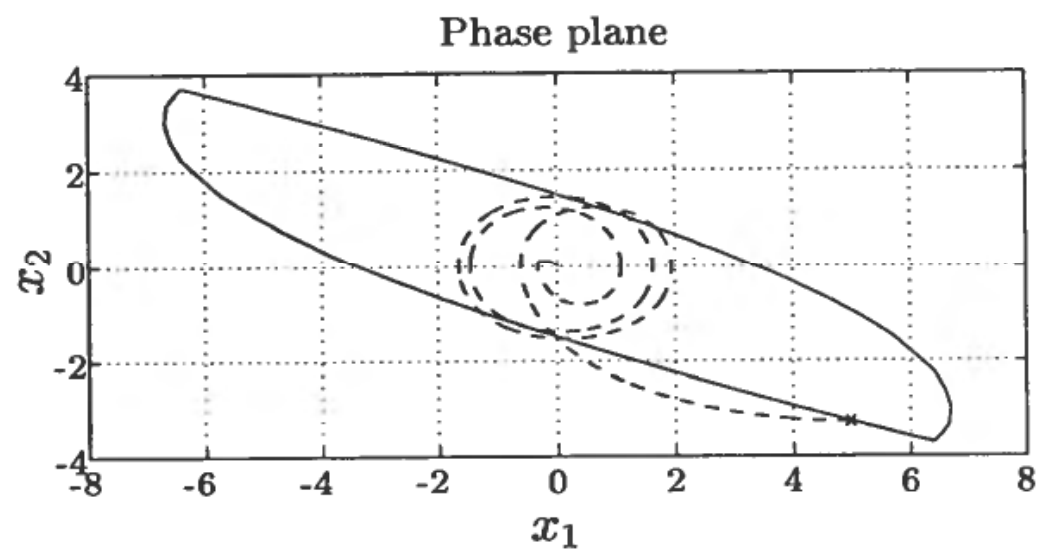

Figure 2. The figure is showing estimates of the sets $\chi$ (solid line) and the time response for $x(0)=[5,-3 \cdot 27]^{T}$ (dashed line).

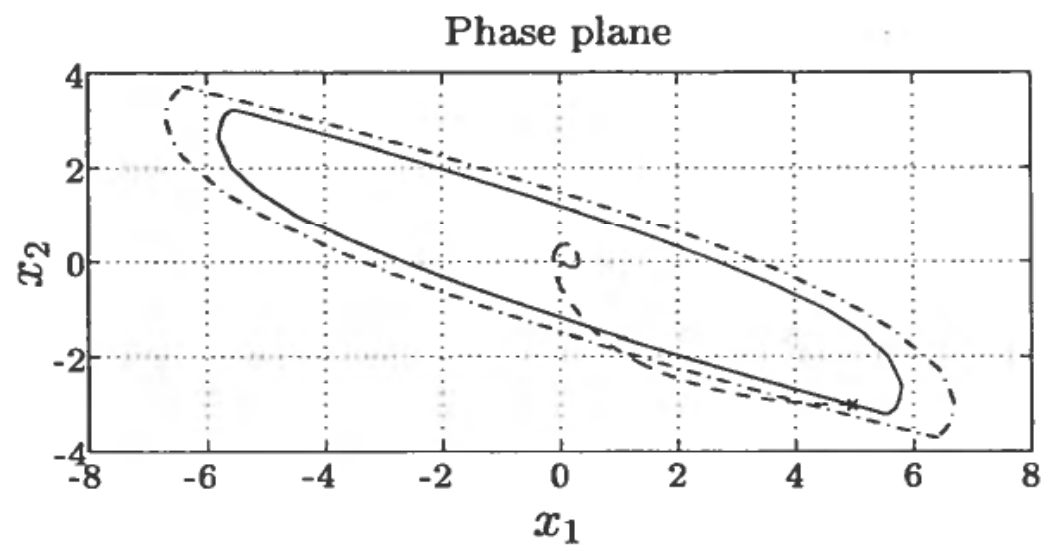

Figure 3. The figure is showing estimates of the sets $\chi$ (dash dotted line) and $\chi_{2.5}$ (solid line). In addition, the time response for $x(0)=[5,-3.03]^{T}$ (dashed line) is shown.

the threshold the oscillations are reduced. Defining the following set of initial conditions

$$
\chi_{\Delta T} \triangleq\left\{x\left(t_{0}\right) \in \mathbb{R}^{n}: h(t) \leqslant \Delta T, t \geqslant t_{0}\right\}
$$

Taking $\Delta T=2 \cdot 5$, an estimate of the set $\chi_{2 \cdot 5}$ is shown in Figure 3 (solid line) together with $\chi$ (dash dotted line). The improved performance is illustrated by the time response for $\boldsymbol{x}(0)=[5,-3 \cdot 03]^{T} \in \chi_{2 \cdot 5}$, shown in Figure 3 (dashed line).

Remark 3. It is important to notice that by using the proposed algorithm for gain reduction, the input is allowed to rate saturate. This clearly gives the possibility of better utilization of the available control authority.

Remark 4. Although $h(t)$ is very useful in detecting possible instability and unsatisfactory performance on-line, it is not suitable for stability analysis in the usual sense since it is not clear how to calculate the set $\chi_{\Delta T}$ a priori. 
From Example 2 it is clear that $h(t)$ is well suited to indicate when to reduce $\gamma(t)$, and the next question is how to reduce the control gains when $h(t)>\Delta T$. There are several answers to this, but we propose to define $\gamma(t)$ in the following way

$$
\gamma(t) \triangleq\left(\frac{\Delta T}{h(t)}\right)^{p}, \quad \text { if } h(t)>\Delta T
$$

where $p>0$. The time derivative of $\gamma(\mathrm{t})$ can be altered with $p$. In particular, as $p \rightarrow \infty$ the control gains will be decreased instantaneously, while for $p=0$ the controller will remain linear. The definition of $\gamma(t)$ also implies that the control gains are reduced until the instance of time $T_{1}$ when $u\left(T_{1}\right)=u_{c}\left(T_{1}\right)$ and thus $h\left(T_{1}\right)=0$. Moreover, it is clear that for any $M<\infty$ implying $\left\|x\left(t_{0}\right)\right\|<M$ there exists a $p^{*}=p^{*}(M)$ such that for $p \geqslant p^{*}$ the commanded input will satisfy the input constraints for $t \geqslant T_{1}$. This follows from the fact that

$$
\lim _{p \rightarrow \infty} \gamma\left(T_{1}\right)=0
$$

Hence, by proper selection of $\Delta T$ and $p$ we have obtained a controller such that the closed-loop system locally satisfy the performance criteria and has a domain of attraction which can be made arbitrary large.

The above control design will result in slow system response when $x\left(t_{0}\right)$ is such that $h(t)>\Delta T$. This is because a large $p$ will give small control gains at time $T_{1}$. Hence, in order to obtain satisfactory performance also in this case, it is desirable to pick $p<p^{*}$. In this case it cannot be guaranteed that $u=u_{c}, t \geqslant T_{1}$, and we propose to reduce the gains according to

$$
\gamma(t)=\left(\frac{\Delta T}{h(t)}\right)^{p}, \quad \text { if } h(t)>\frac{\Delta T}{\gamma\left(T_{1}\right)^{1 / p}}
$$

Notice that for $t \in\left[T_{1}, T_{1}+\Delta T / \gamma\left(T_{1}\right)^{1 / p}\right]$ the scaling factor is fixed, i.e. $\gamma(t)=\gamma\left(T_{1}\right)$, while it is continuous for $t \geqslant t_{0}$. This procedure can be repeated and we summarize the scaling factor in the following definition.

Definition 2. Let $h(t)$ be a piecewise continuous function of time given by

$$
h(t)=0 \text { if } u=u_{c}, \quad \dot{h}(t)=1 \text { otherwise }
$$

where $u$ and $u_{c}$ are the actual and commanded input, respectively. Let $T_{k}$ denote the time instance when $u=u_{c}$ for the $k$ th time, $\gamma_{k}=\gamma\left(T_{k}\right), \gamma_{0}=1, N$ a positive number and $g(t)=\left(t-T_{N}\right)$. Define $\gamma(t)$ as

$$
\gamma(t) \triangleq\left\{\begin{array}{c}
\gamma_{k} \quad \text { if } 0<h(t) \leqslant \frac{\Delta T}{\gamma_{k}^{1 / p}} \\
\left(\frac{\Delta T}{h(t)}\right)^{p} \text { if } k \leqslant N \text { and } h(t)>\frac{\Delta T}{\gamma_{k}^{1 / p}} \\
\left(\frac{\Delta T}{g(t)}\right)^{q} \text { if } k>N \text { and } g(t)>\frac{\Delta T}{\gamma_{N}^{1 / q}}
\end{array}\right.
$$

where $p \geqslant 0, q>1$ and $\Delta T>0$. 


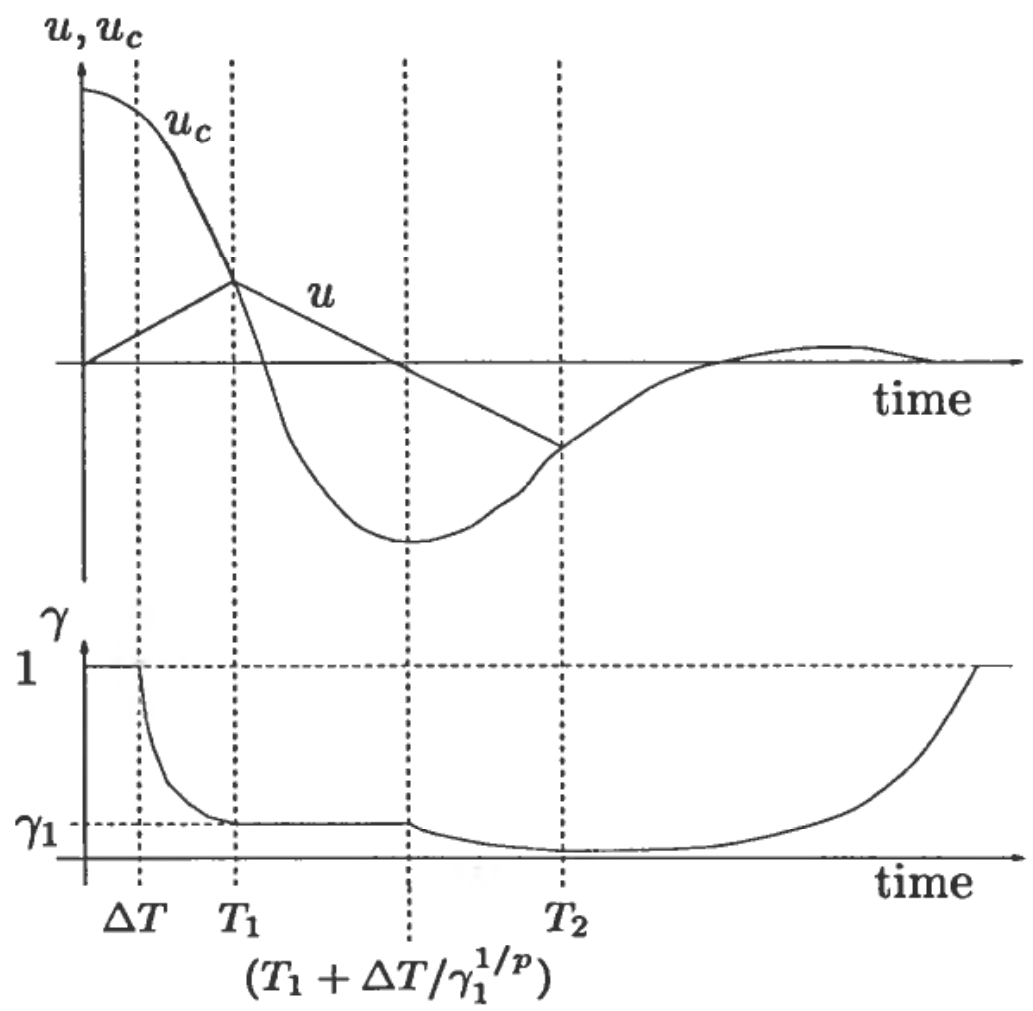

Figure 4. Sketch illustrating the scaling factor when the input is rate saturated.

Remark 5. Notice that $\gamma(t)$ is continuous even though $h(t)$ is discontinuous. Hence, the system (4) with controller (6) and scaling factor (22) is continuous in $t$. Moreover, since it is locally Lipschitz in $\boldsymbol{x}$, there exists a unique solution of the system.

Remark 6. The scaling factor (22) can be explained as follows (see also Figure 4); when the input is saturating, it will, after some finite time $T_{1}$ be equal to the commanded input. If this time is larger than $\Delta T$, the scaling factor $\gamma(t)$ has decreased. Then, if the input does not saturate after this time, convergence follows. However, this is by no means guaranteed, and saturation may occur. Then, after some time $T_{1}+\Delta T / \gamma_{1}^{1 / p}$, the scaling factor will start to decrease until we again have $u=u_{\mathrm{c}}$ at time $T_{2}$. This is repeated a fixed number of times, $N$, and if the input still saturates, $\gamma(t)$ will decrease until it is small enough.

Remark 7. The scaling factor (22) is generalized with the function $g(t)$ and the parameter $q$. The reason for this is to be able to prove convergence of the closed-loop system for any $p \geqslant 0$, see Theorem $1 . k$ will never exceed $N$ when $p$ is properly tuned and typically $k \leqslant 2$ for $p \geqslant 1$.

Based on the scaling factor (22) the following convergence result can be stated.

Theorem 1. Consider the system (4) with controller (6) and scaling factor (22). Then, for any $\beta>0$, any $u\left(t_{0}\right) \in \mathbb{R}$ and any arbitrary large and bounded state initial conditions, $\boldsymbol{x}\left(t_{0}\right)$ the closed-loop system is uniformly convergent, i.e. 
$\lim _{t \rightarrow \infty}\|x(t)\|=0$. Moreover, there exists a time $T$ such that for $\gamma(t)=\gamma(T), t \geqslant T$ we have $u(t)=u_{c}(t), t \geqslant T$.

Proof: See Lauvdal et al. (1997).

From Theorem 1 it is clear that there exists a time $T$ such that $u(t)=u_{c}(t), t \geqslant T$. Thus $\gamma$ has been reduced enough and it is desirable to bring it back to unity value as illustrated in Figure 4. This can be done in numerous ways, but $\gamma$ must be increased slowly. More specifically, when $\gamma$ is increasing we should still have $u(t)=u_{c}(t), t \geqslant T$. One simple approach used in this paper is the discrete formula

where $t_{k}$ is a sampling instance and $\kappa>1$.

$$
\gamma\left(t_{k+1}\right)=\kappa \gamma\left(t_{k}\right)
$$

\section{Simulation Study}

In this section we illustrate some of the features of the gain scheduling approach developed in this paper. Consider an integrator chain of length 3, i.e.

with a linear stabilizing controller

$$
\dot{x}_{1}=x_{2}, \quad \dot{x}_{2}=x_{3}, \quad \dot{x}_{3}=u
$$

where $\boldsymbol{x}_{\text {ref }}$ is the desired equilibrium and

$$
u=-g^{T}\left(x-x_{r e f}\right)
$$

$$
g=\left[\begin{array}{lll}
1 & 3 & 3
\end{array}\right]^{T}
$$

The input is subject to rate saturation of unity value, $\beta=1$. In all of the following simulations $x(0)=0, u(0)=0$ and we consider step inputs in $x_{1}$, that is

$$
\boldsymbol{x}_{\text {ref }}=\left[\begin{array}{lll}
\bar{x}_{1} & 0 & 0
\end{array}\right]^{T}
$$

The rate limit causes the integrator chain with the linear controller (26) to go unstable if $\bar{x}_{1}$ is larger than 1.2 in magnitude.

First, Figure 5 shows a typical time response with the gain scheduled controller
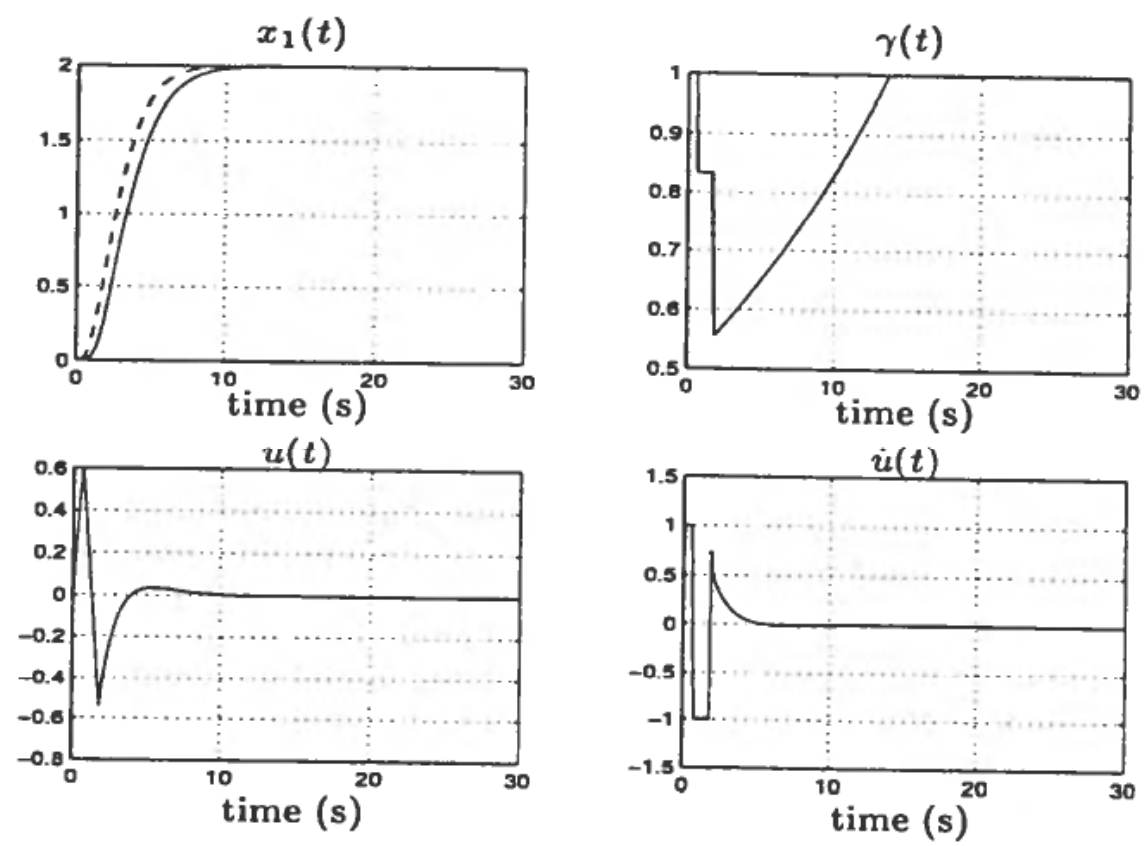

Figure 5. Time response for $\bar{x}_{1}=2, \Delta T=1 / 3$ and $p=1$. 


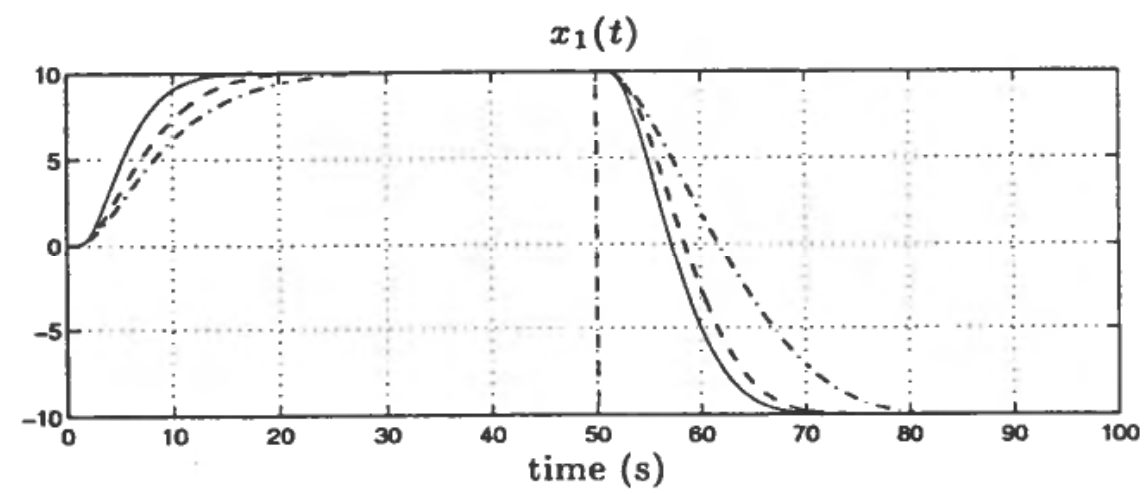

Figure 6. Time response for $\bar{x}_{1}=10, \Delta T=1 / 3$ and $p=1$ (solid line), $p=3$ (dashed line) and $p=10$ (dash dotted line).

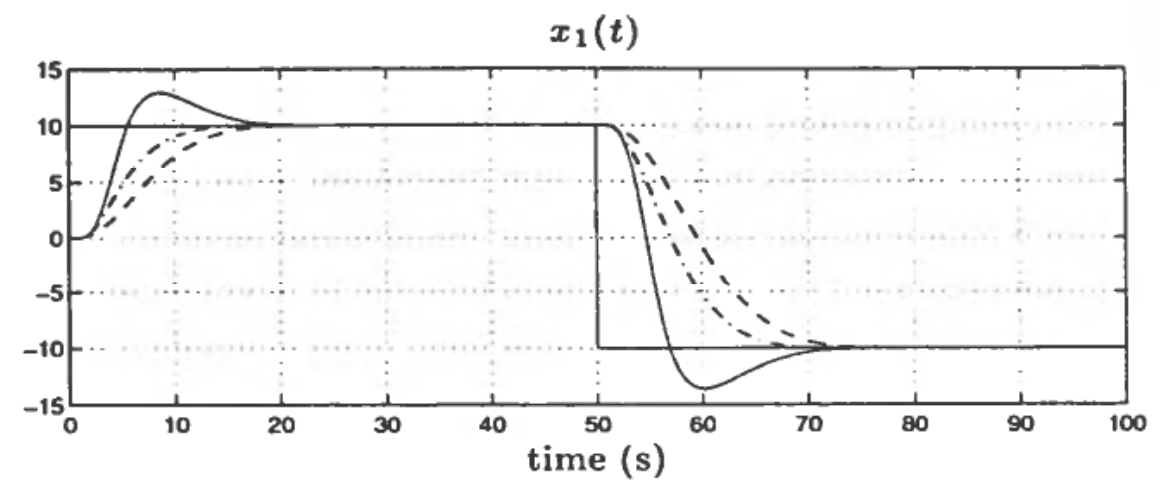

Figure 7. Time response for $\bar{x}_{1}=10, p=1$ and $\Delta T=2 / 3$ (solid line), $\Delta T=1 / 3$ (dash dotted line) and $\Delta T=1 / 10$ (dashed line).

when $\bar{x}_{1}=2, \Delta T=1 / 3$ and $p=1$ (solid line). To illustrate that the performance is very good, the response of the linear controller without the rate limiter (with the rate limiter the system is unstable) is also shown (dashed line). It is seen that the nonlinear control system is just a bit slower than the linear system and does not exhibit 'windup'.

Figures 6 and 7 illustrate the effects of the tuning parameters $p$ and $\Delta T$, respectively. It is seen that large $p$ give slower system response, while large $\Delta T$ give a faster response. Also notice that overshoot occurs if $\Delta T$ is too large.

Figure 8 shows the response for different step inputs, and it is seen that the performance is good for all step sizes.

\section{Ducted Fan Experiment}

In this section we present results from experiments conducted on a model of a thrust vectored aircraft built at Caltech.

\subsection{Ducted Fan Setup}

To allow unlimited travel in the horizontal direction, the aircraft is mounted on a horizontal boom that rotates around a central post, as shown in Figure 9. While the 

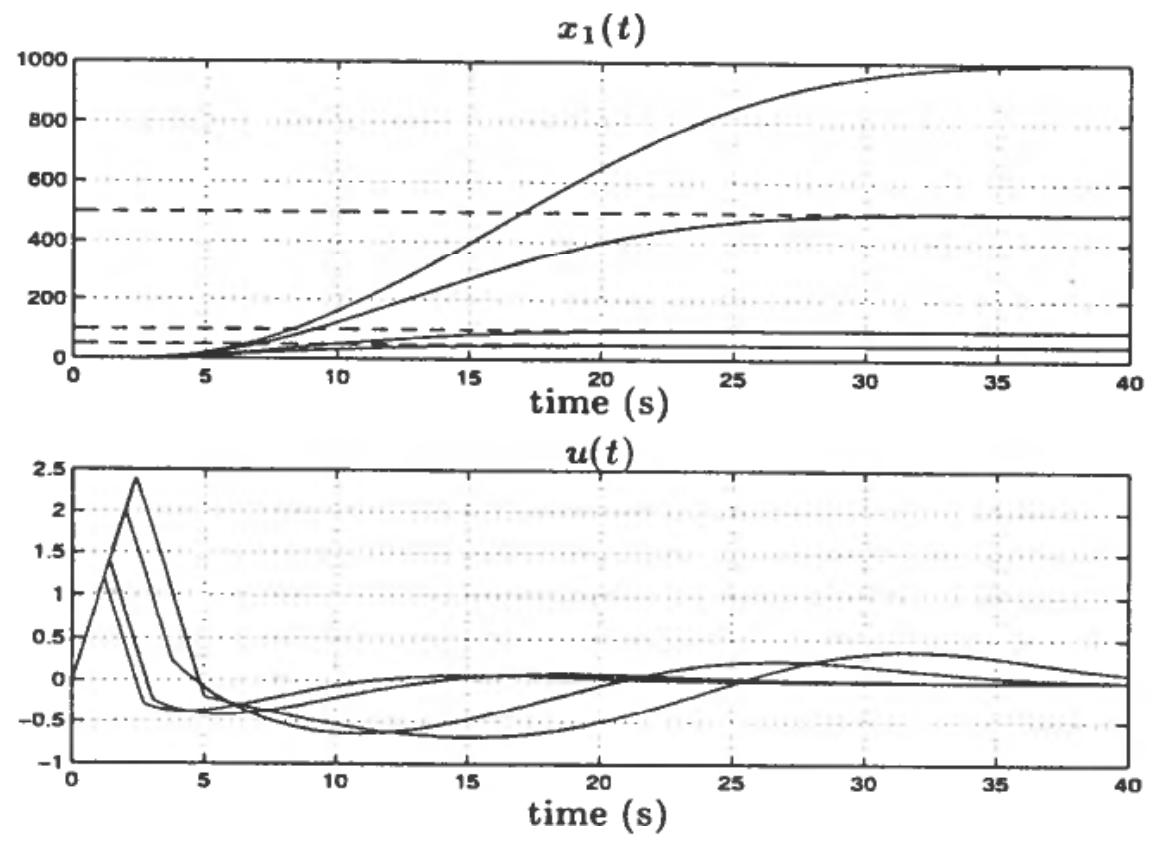

Figure 8. Time response for $\bar{x}_{1}=50,100,500,1000$ and with $\Delta T=1 / 3$ and $p=1$.
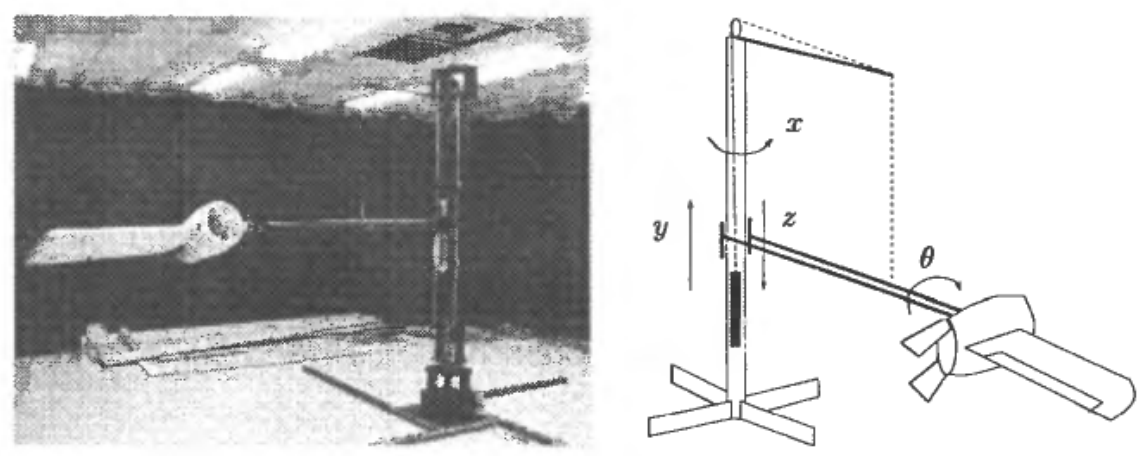

Figure 9. Thrust vectored aircraft on stand.

dynamics of the system do not exactly correspond to chains of integrators, they do approximately correspond to two chains of length two (thrust to longitudinal position) and length four (elevator to pitch to altitude). We apply the controllers of the previous section only to the elevator to altitude control loop.

The aircraft is interfaced to an Intel $48666 \mathrm{MHz}$ PC. It uses a linear current amplifier to regulate the current to a 200 Watt electric motor, driving a propeller. PWM servos to steer the thrust vectoring paddles, and the elevator. The pulse width modulated signal is generated by a custom built PC board. The airfoil is a NACA 0015. Horizontal, vertical and pitch position are measured with encoders. Velocities are obtained by numerical differentiation and smoothing.

The gravitational mass of the fan is offset by a counterweight in the center of the central post, to allow the $200 \mathrm{~W}$ motor to lift the weight of the fan and the boom in 
hover. To reduce the intertial mass in the vertical direction, this counter weight is attached through a pulley with gear ratio $1: 5$. This results in different gravitational masses $\left(m_{g}\right)$, and intertial masses in the $x$ and $z$ direction respectively $\left(m_{x}\right.$ and $\left.m_{z}\right)$.

The ducted fan is interfaced to PC-based data acquisition and control systems and controlled using the Sparrow real-time kernel (for documentation, see Murray et al., 1995). The computer is a standard Pentium based system with commercial digital/ analog converters (for controlling the motor amplifier) and a custom made digital interface board for the PWM outputs to the R/C servos and quadrature encoder inputs from the stand joint encoders Sparrow is a collection of programs and a library of $\mathrm{C}$ functions intended to aid in the implementation of real-time controllers on PCbased data acquisition and control systems. It contains functions for executing control algorithms at a fixed rate, communicating with hardware interface cards, and displaying data in real-time.

\subsection{Experimental Results}

Based on simulations and actual testing on the plant, the following controller vector were designed,

$$
g^{T}=\left[\begin{array}{llll}
-1 & 0 \cdot 6 & -1 & 0 \cdot 3
\end{array}\right]
$$

where the states to which this control is applied are given by $(\theta, z, \dot{\theta}, \dot{z})$ and the commanded input is the elevator angle. The reference trajectory used in all the experiments is step inputs in the desired altitude of the fan. At time 10 (s) the reference is changed from $-0.2(\mathrm{~m})$ to $-0.6(\mathrm{~m})$, and at time $40(\mathrm{~s})$ it is reset to $-0.2(\mathrm{~m})$. The response of the ducted fan with controller (28) and without any other input limitations than the (negligible) physical limits, is shown in Figure 10.

Next, a rate saturation element was introduced in the software such that the time
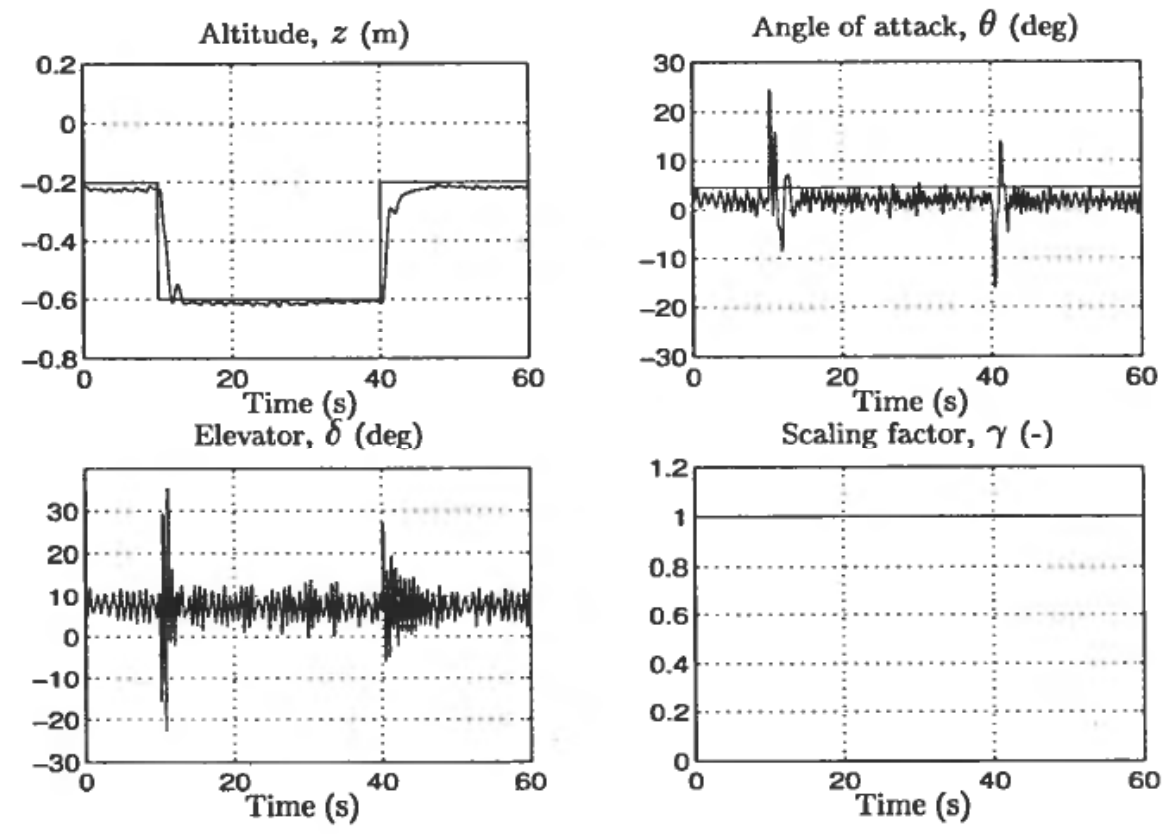

Figure 10. No rate limits. 

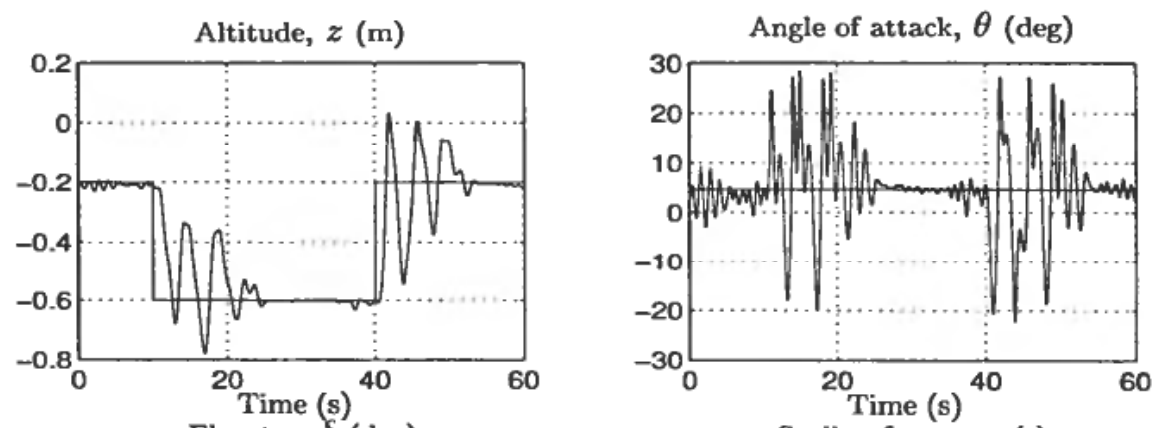

Elevator, $\delta$ (deg)
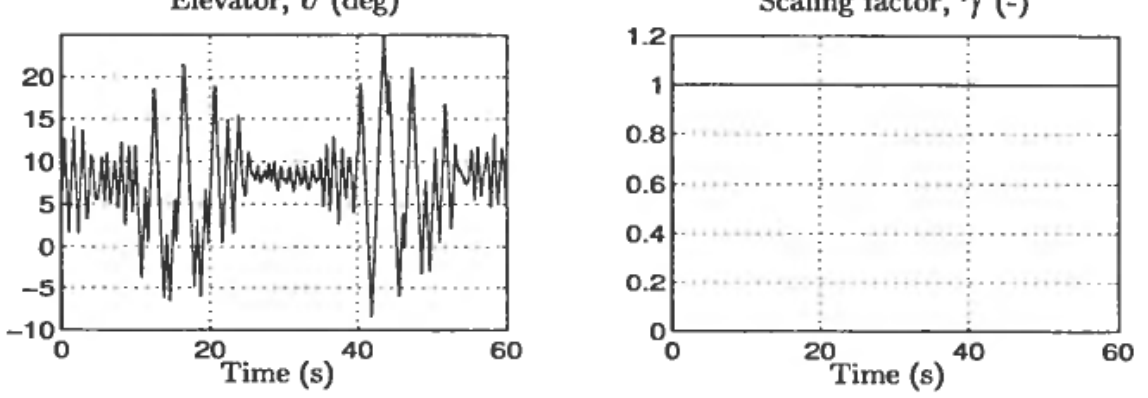

Figure 11. Rate limit; $20(\mathrm{deg} / \mathrm{s})$. No scaling of input.

derivative of the elevator never exceeds a certain value, $\dot{\delta}_{\max }$. The response of the fan with the linear controller and with $\dot{\delta}_{\max }=20(\mathrm{deg} / \mathrm{s})$ is shown in Figure 11. As expected, it is seen that the rate limit leads to a drastic degradation in performance. The altitude $z$ and angle of attack $\theta$ oscillates before they converge to their desired values. This behavior is caused by the rate saturation and can be seen from the plot of the elevator, $\delta$.

In order to reduce the performance degradation caused by rate saturation, we apply the gain scheduling algorithm presented above to the linear controller. As mentioned, the plant can very roughly be approximated to be a chain of four integrators, and the gain scheduled control law is given by

$$
\boldsymbol{g}^{T} \boldsymbol{K}(\gamma)=\left[\begin{array}{llll}
-\gamma^{4} & 0 \cdot 6 \gamma^{2} & -\gamma^{3} & 0 \cdot 3 \gamma
\end{array}\right]
$$

The gain scheduling algorithm of Definition 2 is used, but $h(t)$ is redefined and is given by

$$
h(t)=0 \text { if }\left|u-u_{c}\right| \leqslant \Delta, \dot{h}(t)=1 \text { otherwise }
$$

where $\delta, \delta_{\mathrm{c}}$ is the real and commanded elevator angle, respectively. The reason for introducing $\Delta>0$ is that in a physical system, it is unrealistic to require $\delta \equiv \delta_{c}$. Thus, $\delta$ and $\delta_{c}$ are considered to be equal when $\left|\delta-\delta_{c}\right| \leqslant \Delta$. In order to bring $\gamma(t)$ back to unity value, let

$$
\gamma\left(t_{k+1}\right)=\kappa \gamma\left(t_{k}\right) \text {, if } h(t)=0 \text { and } \gamma_{n}<1
$$

where $t_{k}$ is a sampling instance and $\kappa>1$. In the experiments below the parameters are given by

$$
\Delta T=0 \cdot 2, \quad \Delta=0 \cdot 2, \quad \kappa=1 \cdot 0001
$$



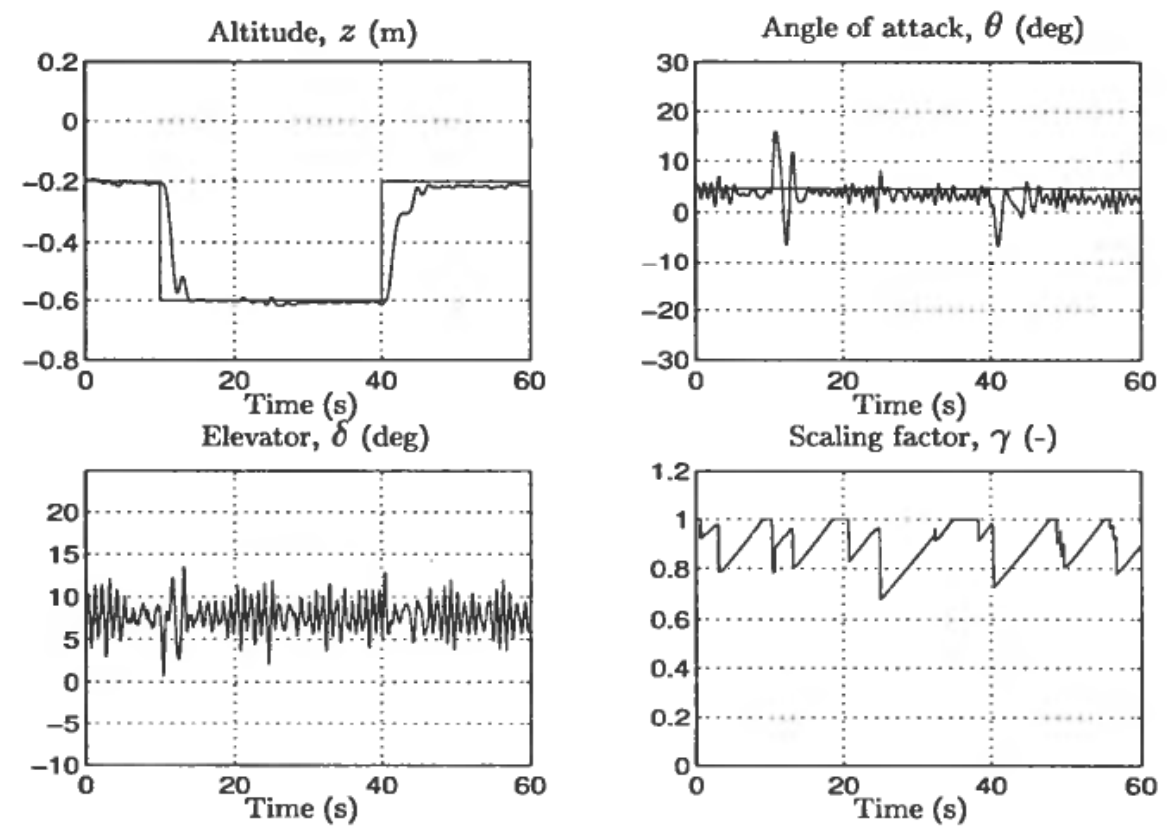

Figure 12. Rate limit; 20 (deg/s). With input scaling.

The response of the ducted fan with the gain scheduled control law when $\dot{\delta}_{\max }=20$ $(\mathrm{deg} / \mathrm{s})$ is shown in Figure 12. Comparing this with the response obtained with the linear controller (Figure 11), it is clear that the performance is very much improved. The altitude $z$ and the angle of attack $\theta$ converge to the desired values without the oscillations obtained with the linear controller. Moreover, the raise time is small and comparing Figure 12 with Figure 10, it is seen that the performance is close to what was obtained with no input limitations.

To illustrate the robustness to different rate limits, $\dot{\delta}_{\max }$ was reduced to $10(\mathrm{deg} / \mathrm{s})$. The response of the linear controller is shown in Figure 13, and it is seen that the performance has worsened and the control system is close to being unstable. The response with the gain scheduled control law derived above is shown in Figure 14. It is important to notice that no tuning was done in this trial and the parameters are given by (32). From the figure is seen that also in this case the performance is satisfactory, even though there is a small overshoot in the step response at $t=40$ (s). However, compared to the linear controller, the improvement is considerable.

\section{Conclusions}

In this paper we have given an algorithm for stabilizing a chain of integrators in the presence of rate saturation. The chief advantage of the algorithm over other approaches described in the literature is that it gives good performance and is not overly conservative. This is verified in experiments on the Caltech ducted fan. The same experiments also indicate that the control system is robust to unmodeled dynamics and disturbances.

This paper is a first step towards a broader goal of developing 'anti-saturation' controllers which compensate for magnitude and rate saturation of actuators in feedback loops while maintaining stability. Extension of the controller to stable plants will 

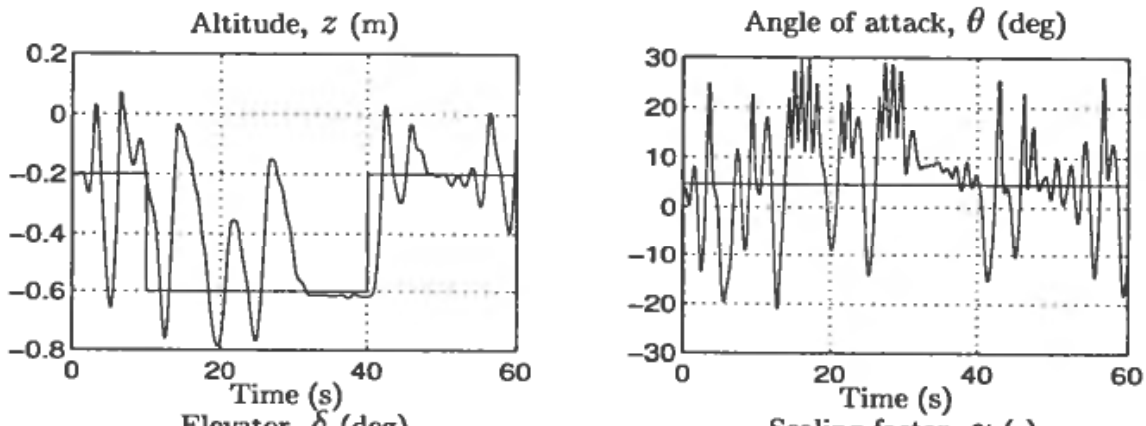

Elevator, $\delta$ (deg)
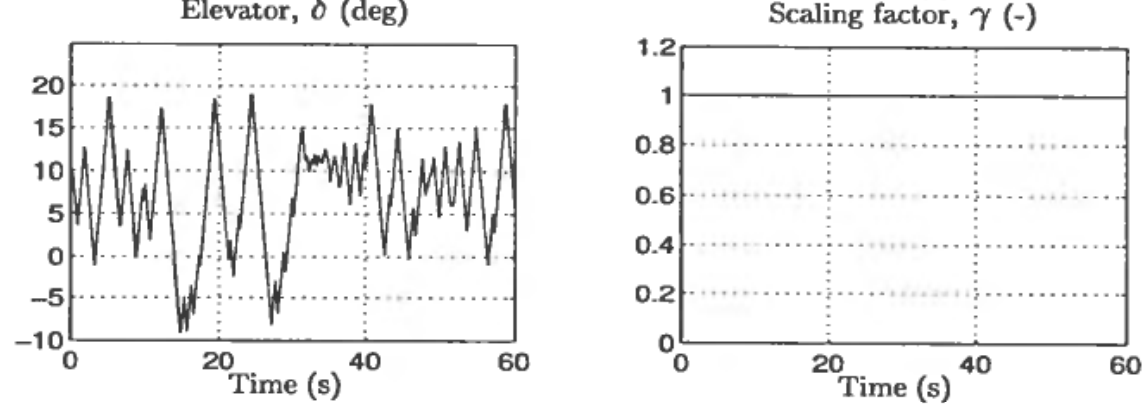

Figure 13. Rate limit; 10 (deg/s). No scaling of input.
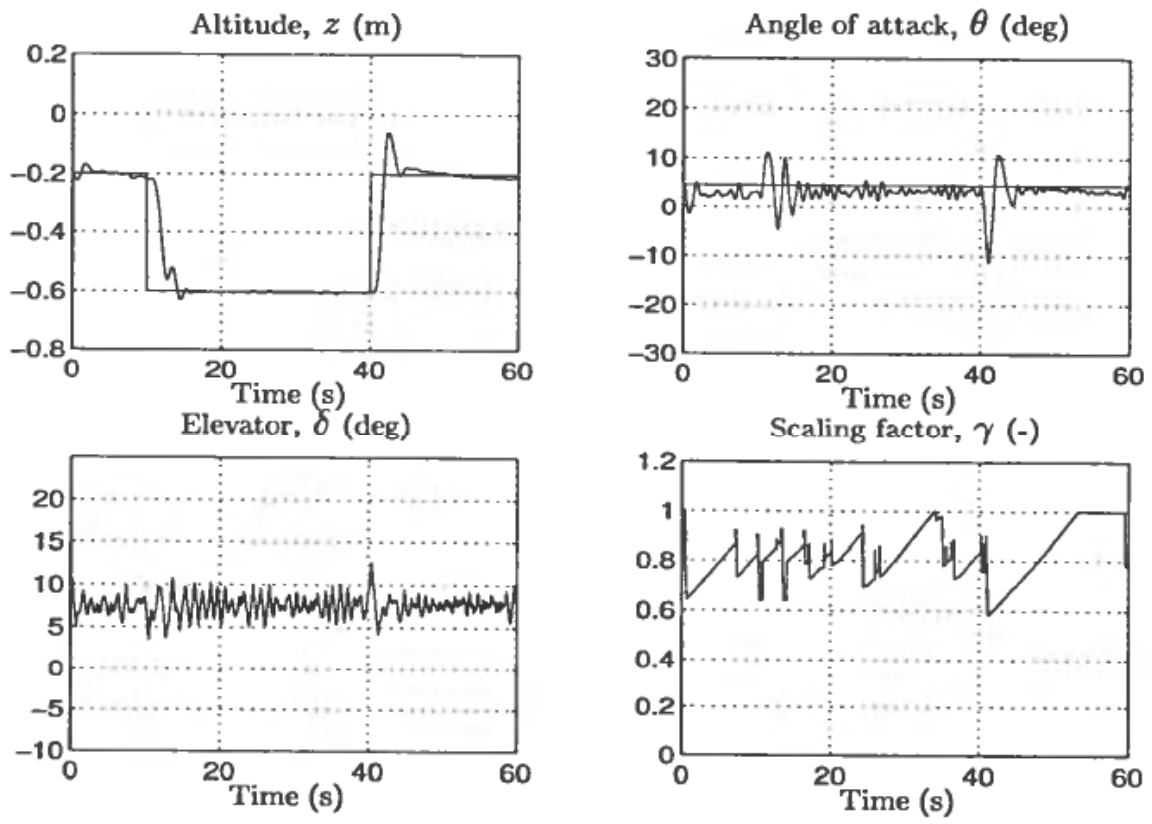

Figure 14. Rate limit; 10 (deg/s). With input scaling. 
be addressed in near future. Future work will also concentrate on giving provable performance for the control laws as well as building more general tools for dealing with saturation.

\section{ACKNOWLEDGEMENTS}

The authors would like to thank Lennart Andersson and Francesco Bullo for their help on this project.

\section{REFERENCES}

BuchHolz, J. J. (1993). Time delay induced by Control Surface Rate Saturation. Z. Flugwiss. Weltraumforsch, 17, pp. 287-293.

DornheIM, M. A. (1992). Report Pinpoints Factors Leading to YF-22 Crash. Aviation Week and Space Technology, November.

Dahleh, M. A. and Pearson, J. B. (1987). $L_{1}$-Optimal Compensators for Continuous-Time Systems. IEEE Transaction on Automatic Control, 32(10), pp. 889-895.

Dahleh, M. A. and Diaz-Bobillo, I. J. (1995). Control of Uncertain Systems: A Linear Programming Approach, Prentice Hall.

BOYD, S. P. and BARRATT, C. H. (1991). Linear controller design: limits of performance, Prentice-Hall.

TeEL, A. R. (1992a). Feedback Stabilization: Nonlinear Solutions to Inherently Nonlinear Problems. PhD thesis, University of California, Berkeley.

TeEL, A. R. (1992b). Global Stabilization and Restricted Tracking for Multiple Integrators with Bounded Controls. Systems \& Control Letters, 18, pp. 165-171.

MeGreTSK1, A. (1996). $L_{2}$ BIBO Output Feedback Stabilization with Saturated Control. IFAC World Congress.

Lauvdal, T., Murray, R. M. and Fossen, T. I. (1997). Stabilization of Integrator Chains in the Presence of Magnitude and Rate Saturations, a Gain Scheduling Approach. The 36th IEEE Conference on Decision and Control (CDC'97). (Submitted).

LAUVDAL, T. and Fossen, T. I. (1997). Semi-Global Results on Stabilization of Linear Systems With Input Magnitude and Rate Saturation. Proceedings of the AIAA Conference on Guidance, Navigation and Control, New Orleans, USA.

MurRaY, R. M., WEMHOFF, E. L. and KANTNER, M. (1995). Sparrow 2.lf Reference Manual, California Institute of Technology. 